\title{
Stimulation électrique fonctionnelle et vélo couché : un apport rééducatif et récréatif dans la prise en charge du blessé médullaire
}

\author{
A.Daubigney, C. Azevedo Coste, B. Sijobert, J. Parent, C. Fattal
}

\section{Introduction}

La stimulation électrique fonctionnelle (SEF) des muscles sous-lésionnels du blessé médullaire améliore les capacités cardiorespiratoires [1], limite la déminéralisation osseuse, l'amyotrophie [2], et les comorbidités [3]. Elle est le plus souvent proposée couplée aux ergomètres à bras [4], ou en« hybrid cycling » [5], en milieu hospitalier mais non applicable en extérieur. Un autre mode d'entraînement, peu exploré dans la littérature, possible en extérieur, existe : le «FES BIKE » (vélo couché couplé à la SEF) [6]. Nous proposons donc de l'aborder dans la présente étude, dite de faisabilité. L'objectif est de proposer le «FES BIKE », comme une activité à part entière, associée à une participation au Cybathlon [7].

\section{Matériel, population et méthode}

Un patient (niveau neurologique Th4, AIS A) participe à l'entraînement, d'une durée d'un an, en trois phases : entraînement quotidien à domicile (stimulateur Cephar ${ }^{\circledR}$ ), bihebdomadaire sur vélo amarré (stimulateur BERKEL ${ }^{\circledR} \mathrm{SB}$ ), puis en extérieur (vélo ICETRIKE Adventure ${ }^{\circledR}+\mathrm{SB}^{\circledR}$ ). Des électrodes de surface sont placées sur les quadriceps et ischio-jambiers. Le patron de stimulation est modifié à partir des patrons du $\mathrm{SB}^{\circledR}$. Les indicateurs de jugement concernent la tolérance physique, l'impact psychologique (échelle de Rosenberg et SF 36), fonctionnel (distance parcourue, vitesse moyenne), physique et technique.

\section{Résultats}

Les résultats présentent une amélioration de la qualité de vie (score physique : +17 points, score mental : +11 points), et de l'estime de soi (40/40 contre 36/40). La distance parcourue (de $70 \mathrm{~m}$ à $750 \mathrm{~m}$ ), la vitesse moyenne (de $2,7 \mathrm{~km} / \mathrm{h}$ à $5,6 \mathrm{~km} / \mathrm{h}$ ), l'endurance à la stimulation (de $1 \mathrm{~min} 31$ à $8 \mathrm{~min}$ ) et la fréquence cardiaque maximale sont augmentées. Le temps de récupération est diminué. Une absence de modifications significatives de la VO2max est constatée.

\section{Conclusion ou discussion}

Cette étude suggère des bénéfices psychologiques et fonctionnels. Elle ne concerne qu'un seul patient. Il apparaît pertinent d'étendre l'étude pour obtenir un niveau de preuve plus élevé. Il serait judicieux de travailler sur les possibilités d'endurance pour atteindre l'objectif du loisir. Proposer d'autres modes d'entraînement pourrait être une piste pour améliorer la VO2max. En introduisant le «FES Bike » le kinésithérapeute valorise l'action de la $\mathrm{SEF}$ et propose une activité nouvelle à caractère rééducatif et récréatif. C'est aujourd'hui le seul mode avéré d'activation motrice sous-lésionnelle à caractère fonctionnel, améliorable dans le temps au prix d'un entraînement régulier.

\section{Références}

[1] G.M. Davis, N.A. Hamzaid, C. FornusekCardirespiratory, metabolic, and biomechanical responses during functional electrical stimulation leg exercise: health and fitness benefits Artif Organs, 32 (8) (2008), pp. 625-629

[2] J.C. Baldi, R.D. Jackson, R. Moraille, W.J. MysiwMuscle atrophy is prevented in patients with acute spinal cord injury using functional electrical stimulation Spinal Cord, 36 (7) (1998), pp. 463-469 
[3] D.E.R. Warburton, A. Krassioukov, S. SprouleCardiovascular health and exercise following spinal cord injury Top Spinal Cord Injury Rehabil, 13 (1) (2007), pp. 98-122

[4] M.P. Galea, S.A. Dunlop, R. Marshall, J. Clark, L. ChurilovEarly exercise after spinal cord injury (switch-on): study protocol for a randomized controlled trial Trials (2015), pp. 16-17

[5] A.J. Bakkum, S. De GrootStolwijk 6 Swüste JM, Van Kuppevelt DJ, AURISC Van Der Woude LH, et al. Effects of hybrid cycling versus handcycling on wheelchair-specific fitness and physical activity in people with long term spinal cord injury: a 16 week randomized controlled trial Spinal Cord, 53 (5) (2015), pp. 395-401

[6] J.S. Petrofsky, H. Heaton 3rd, C.A. PhilipsOutdoor bicycle for exercise in paraplegics and quadriplegicsJ Biomed Eng, 5 (4) (1983), pp. 292-296

[7] R. RienerThe cybathlon promotes the development of assistive technology for people with physical disabilities J Neuroeng Rehabil, 13 (1) (2016), p. 49 